\title{
Study of Sigmoid Sinus Variations in the Temporal Bone by Micro Dissection and its Classification - A Cadaveric Study
}

\author{
Anup Singh ${ }^{1}$ David Victor Kumar Irugu ${ }^{1}$ Kapil Sikka ${ }^{1} \quad$ Hitesh Verma ${ }^{1}$ Alok Thakar ${ }^{1}$ \\ ${ }^{1}$ Department of Otorhinolaryngology and Head \& Neck Surgery, All \\ India Institute of Medical Sciences, New Delhi, Delhi, India \\ Int Arch Otorhinolaryngol 2019;23:e311-e316. \\ Address for correspondence David Victor Kumar Irugu, MS, \\ Department of Ear, Nose, and Throat, Teaching block, All India \\ Institute of Medical Sciences, 4th floor, Room no.-4057, Ansari Nagar, \\ New Delhi, 110029, India (e-mail: drdvki2776@gmail.com).
}

\section{Introduction}

The anatomy of the sigmoid sinus (SS) is highly variable, and it is of utmost importance to have detailed knowledge about these variations while performing mastoid surgeries/transmastoid approaches, such as cochlear implantations, approaches to the cerebellopontine angle (CPA), and the infratemporal fossa approach (Fisch type-A) for the excision of glomus jugulotympanicum tumors. $^{1-4}$

(1D) David Victor Kumar Irugu's ORCID is http://orcid.org/0000-0002$1732-6504$

received

February 22, 2019

accepted

March 17, 2019
Introduction Sigmoid sinus (SS) variations have been classified variously in the literature. These classifications suffer from some form of shortcoming from a clinical point of view for their application.

Objective We propose a clinically relevant classification of the SS in relation to the posterior semicircular canal (PSCC) and to the exposure of the presigmoid dural plate. The positioning of the SS was analyzed with reference to the volume of the mastoid and to the level of mastoid pneumatization.

Methods A total of 94 formalin-preserved human cadaveric temporal bones were microdissected to carry out a complete mastoidectomy. The SS, the presigmoid dural plate, and the PSCC were exposed, and the position of the former was analyzed in relation to the latter two in order to classify the position of the SS into three grades.

Results Grade I had the best exposure of the presigmoid dura and of the PSCC, while grade III had the poorest exposure of the presigmoid dura and of the PSCC. Grade I SS was associated with good pneumatization and higher mastoid volumes compared with grades II and III.

Conclusions The SS exhibits considerable anatomic variability. A favorable positioning of the SS is associated with a large mastoid volume and pneumatization. A careful preoperative study of the imaging may help in understanding the positioning of the SS and the safety of various transmastoid approaches.
The aim of the present study was to report the anatomical variations of the SS in cadaveric temporal bones (TBs) by microdissection, and to propose a new classification/grading system of these SS variations that is relevant from a surgical viewpoint. The proposed classification emphasizes the hindrance posed by the SS to the visualization of the posterior semicircular canal (PSCC) as a landmark. The extent of visualization of the PSCC works as a surrogate indicator of the space constraint while carrying out the transmastoid approaches (including the retrolabyrinthine [RL] and translabyrinthine $[\mathrm{TL}]$ approaches). The objectification of the 
space constraint classified in this fashion will help in predicting the surgical restraints while accessing various structures via a transmastoid approach and help with more objective documentation of the intraoperative anatomical structures as correlated with varied SS positions. The secondary aim was to assess the relationship of the SS anatomy with the pneumatization of the TB and with the volume of the mastoid cavity.

\section{Methods}

Cadaveric TB dissections were performed in the surgical skills laboratory of our department after approval from the Ethical Committee of our Institute (File No.F.8-522/A-522/2017/RS (Project code number: A-522; dated September $19^{\text {th }} 2017$ ). These TBs were harvested from unclaimed cadavers and preserved in $10 \%$ formaldehyde for sterilization before dissection. The microdissections were performed using a Leica M320 F12 microscope with LED (Leica Biosystems, Wetzlar, Germany), which has an inbuilt 3-megapixel camera plus a HD video system (Leica microscope) under 6.4 magnification. A Forte 200 electric microdril (Saeshin Precision CO. Ltd. \#93-15 PahoDong, Dalseo-Gu, Daegu, Korea) with 25,000 rpm was used for dissection.

After the removal of the soft tissues from the TB, the dissections were performed following the standard procedure. The TB was fixed in the House-Urban TB holder and, after the identification of the Macewen triangle, a cortical mastoidectomy was performed. Each TB dissection procedure was recorded, and important pictures were taken separately under the microscope for analysis.

The bones were categorized as well-pneumatized or under-pneumatized by the dissecting surgeon intraoperatively, depending upon the cellularity of the mastoid cell system. The SS was classified according to the visibility of the structures viewed parallel to the thinned out posterior wall of the external auditory canal (essentially a viewing axis perpendicular to the cortical bone of the mastoid). The volume of the mastoid cavity was assessed conventionally by filling it with a measured volume of water after blocking the mastoid aditus.

The positioning of the SS was classified into three grades (I, II, and III; favorable, intermediate, and unfavorable, respectively) by Irugu DVK \& Singh A, based on its anatomical location, with special reference to the presigmoid bony plate (defined as the bone between the anterior margin of the SS and the PSCC) and to the dome of the PSCC. The grading of the SS with the previously mentioned reference landmarks is detailed below and is represented in -Fig. $\mathbf{1}$.

A. Grade I (favorable): The SS was not hindering the view of the presigmoid bony plate and of the PSCC. All of these structures were clearly visible with a flat surface and good volume of the mastoid cavity with a wide Trautmann triangle. These factors were considered to impart good accessibility for the RL and TL approaches to the CPA.

B. Grade II (intermediate): Anterior placement of the SS, obscuring the view of the presigmoid bony plate but not of the PSCC. The volume of the mastoid cavity was reduced and the Trautmann triangle became narrower compared with grade I. It imposes some hindrance and eventual risk to the approach to the CPA by the RL approach. The TL approach is preferred in such cases.

C. Grade III (unfavorable): The SS was situated so far anterior as to impede the visualization of the presigmoid bony plate and of the PSCC. The Trautmann triangle was severely narrowed and it was associated with a significant reduction in the volume of the mastoid cavity. It was considered to be an unfavorable situation to approach the CPA through the RL or the TL approaches, and the retrosigmoid (RS) approach is advisable in such cases.

Statistical analysis: The volumetric data of the left and of the right sides was compared by using the student- $t$ test. The comparison of volume among the three grades of the SS (with respect to pneumatization) was performed using the one-way analysis of variance (ANOVA) followed by the Bonferroni test correction for multiple comparisons. The Stata 14.1 (StataCorp, College Station, TX, USA) software was used to analyze the data, and $p<0.05$ was considered statistically significant.

\section{Results}

A total of 94 cadaveric temporal bones were dissected in our surgical skill laboratory, comprising 47 bones each, of right and left sides. A total of 59 bones were well-pneumatized, and the other 35 bones were underpneumatized (-Table 1).

According to the SS grading described above, the distribution of the dissected temporal bones is described in -Table 1. Grade I consisted of 37 bones (39.36\%), of which 16 were right-sided (17.02\%) and 21 were left-sided (22.34\%) bones. Grade II consisted of 39 bones (41.49\%) comprising 23 right-sided (24.47\%) and 16 left-sided (17.02\%) bones. Grade III had 18 bones (19.15\%) comprising $8(8.51 \%)$ right-sided and 10 (10.64\%) left-sided bones. The level of pneumatization between the two sides was statistically comparable.

On comparing the SS grading with the volume of the mastoid cavity, grade I was found to have a mean volume of $6.6 \mathrm{ml}$ (standard deviation [SD] of $0.90 \mathrm{ml}$ ), grade II had a mean volume of $4.4 \mathrm{ml}$ (SD of $0.84 \mathrm{ml}$ ), and in grade III, the mastoid volume was $2.0 \mathrm{ml}$ (SD of $0.69 \mathrm{ml}$ ). The volume difference in different SS grades was statistically significant, with a p-value of 0.001 (-Table 2) (-Fig. 2).

The distribution of the bones regarding the SS grading and pneumatization and its correlation with the volume of the mastoid cavity is described in - Table $\mathbf{3}$ and is graphically represented in -Fig. 3. A clear trend of reduction in the volume of the mastoid cavity with an increase in the $\mathrm{SS}$ grade can be appreciated. Within a particular SS grade, the higher the degree of pneumatization, the higher the volume of the mastoid. The $p$-value for the ANOVA between the SS gradings with pneumatization and the volume of the mastoid cavity was 0.0001 . 


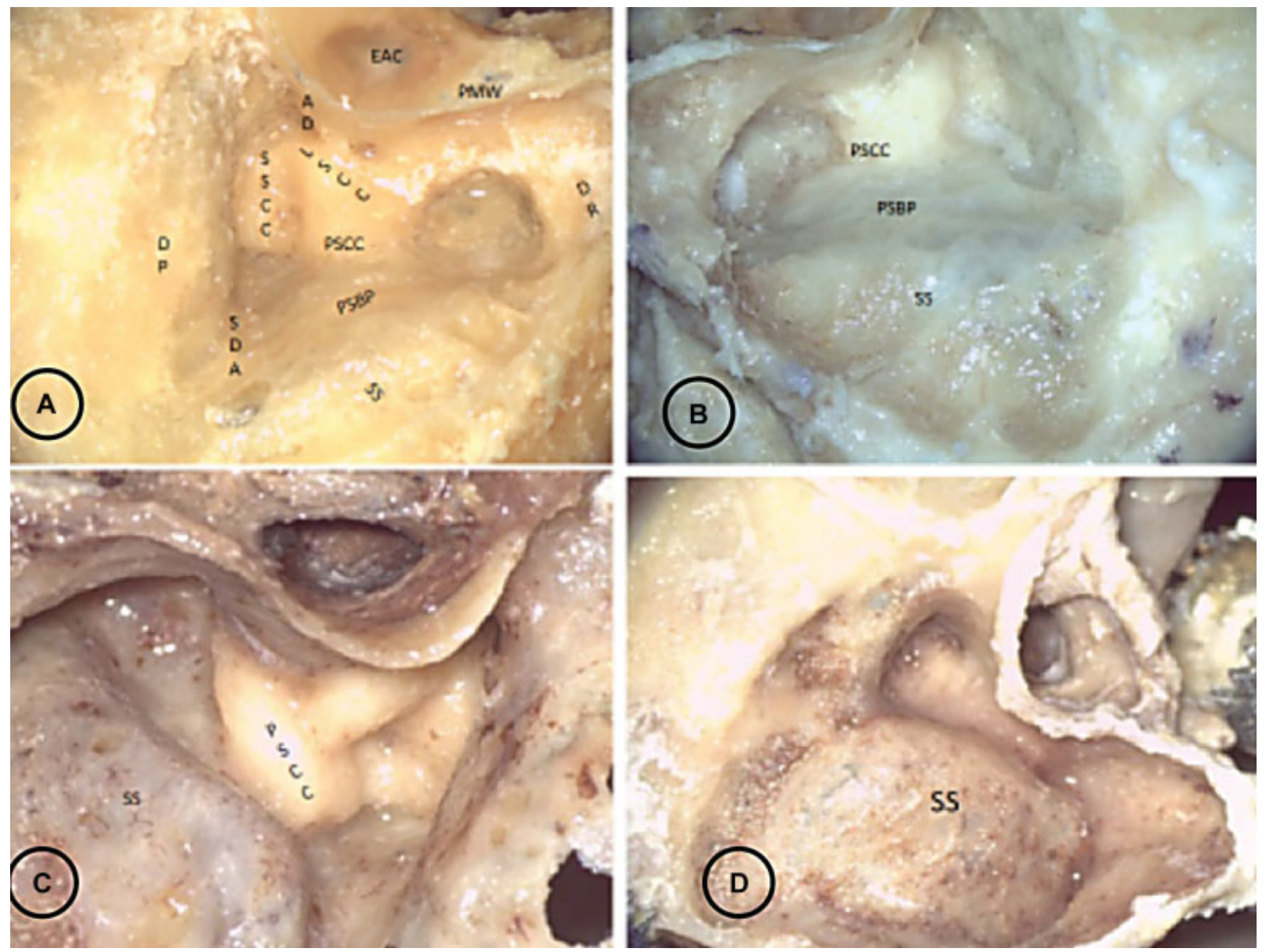

Fig. 1 (A) Dissected temporal bone with marked anatomical reference points for anatomical grading of SS (B) SS grade I: The SS, PSBP, and PSCC are in the same vertical plane. All of the three structures are fully visible with good exposure of the Trautmann triangle and a voluminous mastoid cavity. (C) SS grading II: The SS is anteriorly placed obscuring the PSBP, but the PSCC can be seen. With the anterior placement of the SS, narrowing of Trautmann triangle and the reduction in the volume of the mastoid cavity can be appreciated. (D) SS grading III: Further anteriorly placed SS obscuring the PSBP and the PSCC with a significantly compromised volume of the mastoid cavity and significant reduction of the exposure of the Trautmann triangle. Abbreviations: AD, aditus; DR, digastric ridge; EAC, external auditory canal; LSCC, lateral semicircular canal; PMW, posterior meatal wall; PSBP, presigmoid bony plate; PSCC, posterior semicircular canal; SDA, sinodural angle; SS, sigmoid sinus; SSCC, superior semicircular canal.

Table 1 Distribution of bones according to pneumatization

\begin{tabular}{|l|l|l|l|l|l|}
\hline \multirow{2}{*}{ Pneumatisation } & \multicolumn{3}{|l|}{ Bone side } & \multicolumn{3}{l|}{$\begin{array}{l}\text { Sigmoid sinus } \\
\text { grading }\end{array}$} \\
\cline { 2 - 7 } & Right & Left & I & II & III \\
\hline Well-pneumatized & 31 & 28 & 34 & 23 & 2 \\
\hline Underpneumatized & 16 & 19 & 3 & 16 & 16 \\
\hline Total & 47 & 47 & 37 & 39 & 18 \\
\hline
\end{tabular}

Table 2 Comparative results of sigmoid sinus grading with the volume of the mastoid cavity

\begin{tabular}{|l|l|l|}
\hline $\begin{array}{l}\text { Sigmoid sinus grading } \\
\text { (Irugu \& Singh) }\end{array}$ & $\begin{array}{l}\text { Mean Volume } \\
\text { (SD); (ml) }\end{array}$ & $\begin{array}{l}\text { Number of } \\
\text { bones (\%) }\end{array}$ \\
\hline I & $6.58(0.90)$ & $37(39.4)$ \\
\hline II & $4.41(0.84)$ & $39(41.5)$ \\
\hline III & $1.95(0.69)$ & $18(19.1)$ \\
\hline
\end{tabular}

Abbreviations: $\mathrm{ml}$, milliliters; SD, standard deviation. p-value $=0.001$.

\section{Discussion}

The knowledge of variations in the anatomical location of the SS in the TB is very important for performing mastoid surgeries, especially transmastoid approaches to the CPA. The anatomical variations in the location of the SS affects the exposure of the Trautmann triangle and the volume of the mastoid cavity. ${ }^{1-4}$

The Trautmann triangle is bound superiorly by the superior petrosal sinus, posteriorly by the SS, anteriorly by the PSCC, and anteroinferiorly by the jugular bulb (JB). ${ }^{4-6}$ There have been many attempts to classify the SS in the past based on the location of the SS and on the exposure of the Trautmann triangle, but none of them classify the SS in relation to the surrounding vital structures with consideration to the volume of the mastoid as a function of the position of the SS. The various classifications are listed in - Table 4. ${ }^{1,2,4}$

In 1993, Ichijo et $\mathrm{al}^{7}$ proposed a classification of shapes of the SS. It was a classification of the cross-sectional appearance of the SS based on the study of high-resolution computed tomography (CT) scans of TBs. This classification, 


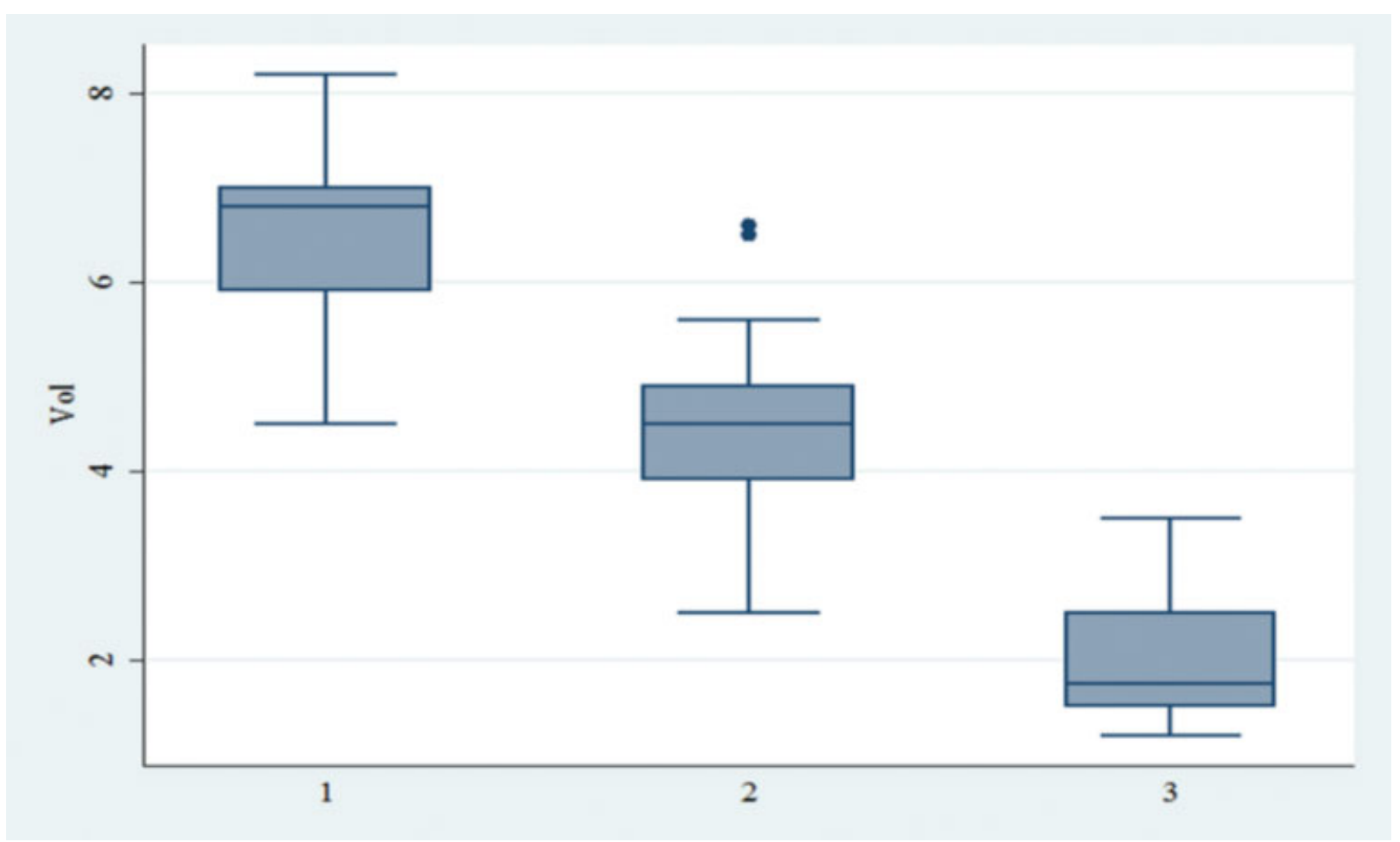

Fig. 2 Box and whiskers plot of sigmoid sinus grading with the volume of the mastoid cavity. ( $x$-axis: sigmoid sinus grade; $y$-axis: volume of the mastoid cavity in $\mathrm{ml}$; Vol: volume). (The boxes represent the interquartile range [the middle $50 \%$ of volume distribution], and the whiskers encompass the entire range of volume distribution. The lines dividing the boxes represent the median, and the dots outside the boxes are the outliers).

Table 3 Distribution of bones with respect to sigmoid sinus grading and pneumatization with the summary of the volume of the mastoid cavity

\begin{tabular}{|l|l|l|}
\hline $\begin{array}{l}\text { Sigmoid sinus } \\
\text { grading and } \\
\text { pneumatisation }\end{array}$ & $\begin{array}{l}\text { Summary of the } \\
\text { volume of the } \\
\text { mastoid cavity; } \\
\text { mean (SD); (ml) }\end{array}$ & $\begin{array}{l}\text { Number of } \\
\text { bones (\%) }\end{array}$ \\
\hline SSG I WP & $6.64(0.91)$ & $34(36.2)$ \\
\hline SSG I UP & $5.93(0.51)$ & $3(3.2)$ \\
\hline SSG II WP & $4.69(0.87)$ & $23(24.5)$ \\
\hline SSG II UP & $4.01(0.62)$ & $16(17.0)$ \\
\hline SSG III WP & $3.25(0.35)$ & $2(2.1)$ \\
\hline SSG III UP & $1.79(0.53)$ & $16(17.0)$ \\
\hline
\end{tabular}

Abbreviations: ml, milliliters; SD, standard deviation; UP, underpneumatized; WP, well-pneumatized.

p-value $=0.0001$.

however, does not provide information about the surrounding structures and is of limited practical importance from an otologic surgical point of view. The classification of Kayalioglu et al has similar limitations. ${ }^{2,8}$

Sarmiento et $\mathrm{al}^{4}$ classified the SS into three types based on its position in relation to the Trautmann triangle. They designated a posteriorly displaced SS as type 1 , which was associated with a large Trautmann triangle. Type 2 and type 3 SSs were anteriorly and medially placed sinus morphologies, associated with a tight Trautmann triangle, more so with the medially placed SS.
In 2009, Sun et al $^{1}$ proposed a radiological classification of the SS in four groups in relation to three lines drawn on CT scan, reflecting important surgical structures. The authors emphasized the ability of the classification system to reflect the SS as a landmark of surgical significance for transmastoid surgeries. However, the radiological data was not backed by the real-time intraoperative dissection findings validating the data.

The position of the SS significantly affects the exposure of the endolymphatic sac via a transmastoid approach, and of the $\mathrm{CPA} /$ petroclival fissure area via a presigmoid retrolabyrinthine approach. ${ }^{5,6}$ The exposure of the posterior cranial fossa via a presigmoid retrolabyrinthine approach depends on the positional relationship of the SS and of the PSCC in an anteroposterior plane and of the Superior Petrosal Sinus (SPS) and of the JB in a superoinferior plane. ${ }^{5,6,9}$ The more forward placed the $\mathrm{SS}$ is, the more constricted the exposure via this corridor gets. This leads to twofold consequences. It impedes the direct access to the area of pathology and risks the surrounding vital structures, including the facial nerve and the posterior labyrinth. Even if the access to the area of concern is obtained, venturing into it via this constrained corridor may hamper maneuvering in the target region and may result in difficulty in achieving complete disease removal, adequate dural closure, and sealing of the cerebrospinal fluid (CSF) leak with the attendant adverse outcomes. 5,6,9

The ideal characteristics of the lesion accessible via this route would be a lesion placed superficially and not based on the fundus of the internal auditory canal, a low JB and an SS placed posteriorly with reference to the posterior 


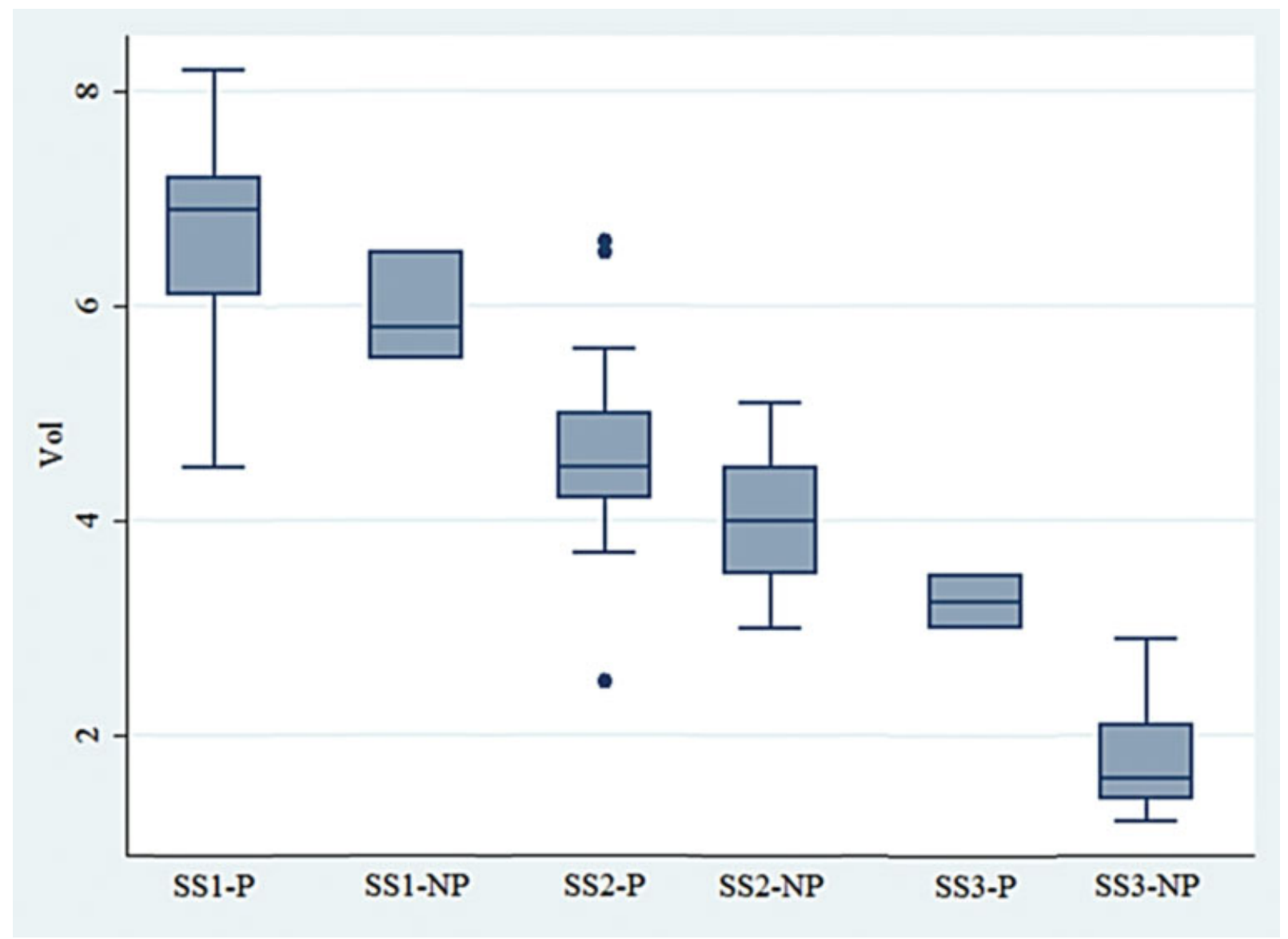

Fig. 3 Box and whiskers plot of the correlation of results between the volume of the mastoid cavity with the sigmoid sinus grading and pneumatization ( $x$-axis: sigmoid sinus groups; $y$-axis: volume of the mastoid cavity in $\mathrm{ml}$ ). (The boxes represent the interquartile range [the middle $50 \%$ of volume distribution] and the whiskers encompass the entire range of volume distribution. The line dividing the box represents the median and the dots outside the box are the outliers). Abbreviations: NP, underpneumatized; P, well-pneumatized; SS, sigmoid sinus; Vol;,volume.

Table 4 Lliterature review of classifications of sigmoid sinus variations

\begin{tabular}{|l|l|l|}
\hline S. No. & $\begin{array}{l}\text { Author } \\
\text { and Year }\end{array}$ & $\begin{array}{l}\text { Basis of the Classification } \\
\text { [reference }]\end{array}$ \\
\hline 1 & $\begin{array}{l}\text { Ichijo et al } \\
(1993)\end{array}$ & Shape of the sigmoid sinus \\
\hline 2 & $\begin{array}{l}\text { Kayalioglu et al } \\
(1996)\end{array}$ & $\begin{array}{l}\text { Anatomical position of the } \\
\text { sigmoid sinus in the } \\
\text { mastoid cavity }{ }^{2}\end{array}$ \\
\hline 3 & $\begin{array}{l}\text { Sarmiento et al } \\
(2004)\end{array}$ & $\begin{array}{l}\text { Based on the narrowing of } \\
\text { the Trautmann triangle }\end{array}$ \\
\hline 4 & $\begin{array}{l}\text { Sun et al } \\
(2009)\end{array}$ & $\begin{array}{l}\text { Three imaginary radiological } \\
\text { (computed tomography) } \\
\text { reference lines theory }\end{array}$ \\
\hline
\end{tabular}

semicircular canal. With the lesion not conforming to these attributes, the surgeon may need to convert to a transcrusal/ translabyrinthine approach, to skeletonization/posterior displacement of the SS, or to undertake a retrosigmoid approach. $^{6,10}$

Through our study, we have proposed to classify the ease of transmastoid approaches according to the location of the SS in relation to the PSCC. We have classified the SS into three categories (grade I, II and III; favorable, intermediate and unfavorable grades, respectively). The most common pattern was that of the grade II SS (41.5\%), followed by grade I SS (39.4\%); grade III being the least common pattern (19.1\%). A grade I SS affords a large exposure of the Trautmann triangle, as well as an ample surgical space to execute various transmastoid approaches. The exposure gets progressively diminished as we move from grade II to grade III SSs. The volume of the mastoid correlates significantly with the location of the SS in TBs. The prediction of the volume of the mastoid cavity was significantly affected by the location of the SS and by the pneumatization of the TB (6.6 ml with well-pneumatized grade I SS versus $1.8 \mathrm{ml}$ in cases of underpneumatized grade III SS; $p=0.0001$ ).

The present study emphasizes the difficulties in performing the surgeries in the distorted anatomy, with special reference to mastoid surgeries, implantation surgeries, and transmastoid approaches, such as endolymphatic sac decompression, CPA tumor excision, and the infratemporal fossa approaches to glomus tumors. Every patient needs to be evaluated preoperatively thoroughly with a suitable radiological method, especially high-resolution $\mathrm{CT}$ of the $\mathrm{TB}$, which provides knowledge of the anatomy and of the extent of the disease involving the $\mathrm{TB}$, to avoid intraoperative complications, as well as to enable better counseling of the patient. ${ }^{11-13}$ 


\section{e316 Study of Sigmoid Sinus Variations in the Temporal Bone Singh et al.}

\section{Conclusion}

1. The present study presents a clinically oriented classification of the positioning of the SS in three grades.

2. Sigmoid sinus grades I, II and III were noted to be present in $39.4,41.5$ and $24.4 \%$ of the cases, respectively.

3. Access to the posterior fossa for the transmastoid, TL and RL approaches is easier and wider in grade I SS.

4. Well-pneumatized bones had a higher probability of having grade I SS (favorable configuration), while underpneumatized bones have a more unfavorable configuration of the SS.

5. The volume of the mastoid impacts the location of the SS. The more favorably placed SSs were found to be associated with a larger volume of the mastoids.

Conflicts of Interests

Irugu D. V. K. reports grants from the All India Institute of Medical Sciences, New Delhi, India, during the conduct of the study; grants from the All India Institute of Medical Sciences, New Delhi, India, outside the submitted work.

\section{References}

1 Sun D, Lee DH, Jang KH, et al. A Suggested New Classification System for the Anatomic Variations of the Sigmoid Sinus: A Preliminary Study. J Int Adv Otol 2009;5(01):1-5

2 Kayalioglu G, Gövsa F, Ertürk M, Arisoy Y, Varol T. An anatomical study of the sigmoid sulcus and related structures. Surg Radiol Anat 1996;18(04):289-294
3 Thamke S, Kalra S, Khandelwal A. Morphometric evaluation of dural venous sinuses: anatomical study with its implications in surgical interventions. J Morphol Sci. 2016;33(02):83-89

4 Sarmiento PB, Eslait FG. Surgical classification of variations in the anatomy of the sigmoid sinus. Otolaryngol Head Neck Surg 2004; 131(03):192-199

5 Tubbs RS, Griessenauer C, Loukas M, Ansari SF, Fritsch MH, CohenGadol AA. Trautmann's triangle anatomy with application to posterior transpetrosal and other related skull base procedures. Clin Anat 2014;27(07):994-998

6 de Melo JO Junior, Klescoski J Junior, Nunes CF, Cabral GA, Lapenta MA, Landeiro JA. Predicting the presigmoid retrolabyrinthine space using a sigmoid sinus tomography classification: A cadaveric study. Surg Neurol Int 2014;30(05):131

7 Ichijo H, Hosokawa M, Shinkawa H. Differences in size and shape between the right and left sigmoid sinuses. Eur Arch Otorhinolaryngol 1993;250(05):297-299

8 Shibghatullah AH, Abdullah MK, Mohamad I. Unusual Superficial Lateral Course of Sigmoid Sinus. 2012;4(01):250

9 Van Beek-King J, McKinnon B. Retrolabyrinthine approach. Operative Techniques in Otolaryngology. 2013;24(03):169-171

10 Brandt MG, Poirier J, Hughes B, Lownie SP, Parnes LS. The transcrusal approach: a 10-year experience at one Canadian center. Neurosurgery 2010;66(05):1017-1022

11 Visvanathan V, Morrissey MS. Anatomical variations of the temporal bone on high-resolution computed tomography imaging: how common are they? J Laryngol Otol 2015;129(07):634-637

12 Karaca CT, Toros SZ, Noseri KH. Analysis of Anatomic Variations in Temporal Bone by Radiology. Int Adv Otol. 2012;8(02): 239-243

13 Jovanović I, Ugrenović S, Stojanović V, Krstić M, Trandafilović M, Čukuranović J. Morphometric characteristics of Jugular foramen and sigmoid sinus groove: their possible connections with high jugular bulb presence. Facta Univ Med Biol. 2014;16(01): $12-17$ 